A Guide-Book to Biochemistry

By Kenneth Harrison. Pp. viii + 150. (Cambridge: At the University Press, 1959.) Cloth edition, 17s, $6 d$. net; Paper edition, $10 s .6 d$. net.

T HERE are several good modern biochemistry books in the thousand-page category, but there is a real need for the introductory type of book of a hundred pages or so. Biochemistry has grown into such a vast subject that an author who is courageous enough to write a small book about it is sure to be criticized for what he decides to leave out, if not for what he puts in. The decision will, of course, depend on the type of reader the author has in mind. Accord. ing to the dust cover (for which the author may not be responsible), this book is aimed not only at science students at the university, but also at boys and girls at school who may be thinking of taking up biochemistry later on. What will these potential biochemists find in the book ? After a short introduction they will find chapters on energy, oxidation (including several pages on the $r \mathrm{H}$ scale), photosynthesis, metabolism of carbohydrates, fats and proteins, and a final chapter on control of metabolism. For good measure they will be able, in an appendix, to study the full structural formulæ of complex molecules such as coenzyme $A$ or uroporphyrin-III. But they will find nothing about, say, hæmoglobin and its functions, and, as the author himself says, it is a far cry from the contents of this book to a dog or a cat or even a cabbage. One wonders whether the cry need have been quite so far as it is. No, the value of this book is not so much as an introductory guide to biochemistry for school children or for university students, but as a means of helping students to consolidate and summarize biochemical knowledge which they have gained from their lectures and text-books. Here it will fulfil a most useful purpose. The information, though so highly condensed, is clearly set out and is up to date. The book is well produced, and few misprints were noticed. The price is very moderate, and it was a good idea to make available a paperbacked edition at an even lower price.

D. C. HarRISON

\section{Nuclear Electronics}

Proceedings of the International Symposium on Nuclear Electronics organized by the French Society of Radioelectricians. (Sessions I-5.) Pp. xii +452 . (Vienna: International Atomic Energy Agency, 1959.) 84 schillings ; $24 s . ; 4$ dollars.

THIS volume contains the texts of fifty papers presented by delegates from the European countries and the United States at sessions 1-5 of the symposium on nuclear radiation detectors, associated pulse techniques and reactor control measurements and instrumentation. About a half of the papers are printed in English and the rest in French. A brief record of the discussion periods is given at the end of each of the five sections.

Session 1, on scintillation radiation detectors, includes a general review paper on the characteristics of phosphors and photo-multipliers developed during the past ten years and summarizes the important problems for future investigation. Separate papers deal with fast photo-multiplier tubes and with scintillation processes in phosphors, including variation of phosphor decay-time with specific ionization, and its application in differentiation of particle types. Session 2 is devoted to other types of radiation detectors used mainly in nuclear physics measurements, and includes short papers on ionization counters, scintillation in gases, a gas Cerenkov counter and semi-conductor radiation detectors. Three papers describe developments in gamma-ray spectrometry.

Session 3, on fast pulse techniques, includes a brief review of fast electronics in high-energy physics, and separate papers on trigger circuits, coincidence circuits, timing circuits and pulse generators for use in the nano-second time region. Session 4 on pulse techniques is devoted mainly to pulse-height analysers while Session 5 contains a short review on philosophy of reactor safety control in the United States, and several contributions on control systems and measurements in French reactors.

Much of the information has been published elsewhere in greater detail, but the collected papers will serve as a useful reference book on recent developments to designers and users of nuclear electronic equipment.

W. ABSON

\section{Electromagnetic Radiation from Cylindrical Struc- tures}

By James R. Wait. Pp. ix +200 . (London and New York: Pergamon Press, 1959.) 50s. net.

$r$ HE designer of aerials for aireraft and missiles must co-operate with the aerodynamicist and is therefore led to types of aerial which can be mounted on wings or fins without disturbing their aerodynamic properties. A satisfactory form of aerial is the slot. for which the behaviour in an ideal infinite conducting plane is well known. On an aircraft, however, the slot must be mounted on a relatively small conducting surface, which inevitably has an appreciable curva. ture. There is therefore a need to have information on the influence of curved surfaces on the radiation from slots and also from small dipoles mounted at right angles to the surface. The author of the present book has tackled this problem for those surfaces which admit the possibility of a formal electromagnetic solution. The surfaces include circular and elliptic cylinders, wedges and corner reflectors. In each case a detailed mathematical solution, usually in series form, is derived and comprehensive numerical results are given for the parameters likely to be of practical interest. These numerical results, which are given graphically, can be readily used by the practical designer even if he is not interested in the details of the theory.

The material is elearly presented and should be intelligible to the physics or electrical engineering graduate with a reasonable knowledge of electromagnetic theory. A useful appendix on the classical theory of radiation from current distributions serves as a revision of the fundamental results from which the main text is developed. An excellent feature is the frequent reference by the author to the similarity between radiation and seattering problems, and the reciprocity theorem is used to illustrate this. The volume is thus also of value to those interested in the theory of electromagnetic wave scattering. In particular, the short account of the work of Fock in this field provides an admirable introduction to the many original papers which use Fock's methods.

This book fills a gap in the existing literature and is strongly recommended to those with theoretical interests in aerials and electromagnetic scattering problems. 